\title{
INTEGRACIÓN DE TECNOLOGÍA A TRAVÉS DEL AULA VIRTUAL Y EL CAMBIO EN PRÁCTICAS PEDAGÓGICAS DE DOCENTES
}

\section{Introducción}

Uno de los grandes desafíos de la educación superior en Chile es la innovación académica, que significa un cambio en las prácticas pedagógicas de los docentes universitarios. Las Tecnologías de la Información y Comunicación (en adelante TIC) son algunos de los recursos que se pueden emplear, sin embargo no suponen por sí mismas una garantía de cambio positivo en la universidad. Disponer de nuevos recursos que permitan nuevas formas de hacer las cosas no significa que necesariamente se produzca el cambio (Marquès, 2001).

Es así como la Universidad del Pacífico puso a disposición de los docentes una plataforma virtual para apoyar la clase presencial, sin embargo el interés inicial por utilizarla fue escaso. Contar con la herramienta no fue suficiente, se requería una estrategia de implementación. Así surgió un plan estratégico para estimular su uso, que consistió en mostrar, motivar, capacitar, experimentar y retroalimentar.

El presente artículo se organiza en cuatro secciones. La primera presenta algunos antecedentes de la experiencia, la segunda la implementación del programa, la tercera, algunos cambios observados en los docentes, y la cuarta entrega algunos datos respecto a la relación de los estudiantes con la experiencia, finalizando con algunas reflexiones generales.

\section{Antecedentes}

El modelo educativo de la Universidad del Pacífico reconoce las diferencias de sus estudiantes y centra el aprendizaje en ellos. 
Asimismo, se caracteriza por la promoción de tres prácticas pedagógicas:

- El desarrollo de ambientes de aprendizaje y estrategias didácticas que fortalezcan la colaboración, el trabajo en equipo y la innovación.

- El uso de metodologías activas y de la tecnología como recurso didáctico.

- La promoción de la flexibilidad pedagógica a través de escenarios de aprendizaje fuera de la clase, en prácticas integradas y en aulas virtuales.

En este contexto, semestralmente se habilita un aula virtual en todas las asignaturas de pregrado, y su uso es libre y voluntario, tanto en la estructura como en el modo de emplearla. En ella tienen la posibilidad de planificar y organizar actividades pedagógicas, para que el estudiante desarrolle en forma autónoma, interactiva y asincrónica, en función del logro de algún objetivo del programa o para retroalimentar un tema tratado en la clase presencial.

La herramienta que se utiliza es Moodle, que corresponde a una plataforma de aprendizaje, de uso libre, con una interfaz de navegación sencilla y eficiente. Desde el punto de vista pedagógico, permite agregar recursos como textos, presentaciones, videos, programas, sitios web y actividades como consulta, tarea, chat, foro, glosario, Wiki, talleres, lección, cuestionario, entre otros.

\section{Implementación del proyecto}

Existe evidencia en la bibliografía especializada respecto a las resistencias y dificultades con que se suelen enfrentar los proyectos innovadores en materia de incorporación de TIC en el campo educativo. Salinas (2002) señala que "es obvio que las instituciones encuentran, al mismo tiempo, dificultades de diversa índole para la incorporación de las TIC. Entre éstas: falta de estrategia institucional, fuerte resistencia del personal académico y administrativo y, a veces, falta de previsión de los costes implicados. Parece, pues, conveniente que cada institución formule una visión conjunta de futuro sobre el modelo propio de enseñanza y aprendizaje". No fue distinto en el 
caso de este proyecto, así el primer obstáculo que se presentó fue la resistencia por parte de los directivos y docentes para utilizar la plataforma; algunas de sus causas fueron: actitud negativa hacia la tecnología, falta de aporte económico extra, carencia de competencias tecnológicas y tiempo que deben dedicar a esta actividad. No obstante lo anterior, el proyecto se implementó utilizando la siguiente secuencia: mostrar-motivar, capacitar, experimentar y retroalimentar:

\subsection{Primera etapa: mostrar-motivar}

Se inició bajo la condición de no obligar, ni imponer, sino más bien estimular y encantar. Lo primero fue difundir la existencia del aula virtual entre los docentes. Se confeccionó un afiche de presentación, que se distribuyó en todas las salas de profesores de cada sede y también fue enviado al correo electrónico personal.

Luego, para despertar el interés de los académicos se utilizaron distintas estrategias para mostrar cómo usar dichas aulas. Entre ellas se creó la "Red de Colaboración", integrada por los docentes que impartían clases en el semestre, los que fueron invitados a la presentación de las aulas virtuales que estaban funcionando para observar cómo era el uso de éstas en otras asignaturas. De este modo, muchos de ellos participaron por curiosidad, en algunos casos como primer acercamiento, y conocieron en qué consistían.

Finalmente se realizaron jornadas de sensibilización, donde se reflexionó sobre las exigencias de la sociedad actual en la educación superior respecto al cambio de rol del estudiante y del docente, presentando el aula como un recurso que facilita el cambio y responde a las exigencias de formación de los futuros profesionales del siglo XXI.

\subsection{Segunda etapa: capacitación}

Una vez que se dio a conocer este recurso, se inició el proceso de familiarizar a los docentes con la plataforma, a través de talleres de capacitación. Los recursos y actividades que se trabajaron durante la jornada se desarrollaron en torno a las preguntas: ¿qué?, ¿para qué? y ¿cómo? De esta manera, los profesores conocieron y aplicaron inmediatamente el aula virtual de la asignatura que impartían. Esto 
les permitió descubrir la utilidad y los motivó a continuar practicando en forma autónoma.

Para conocer la impresión de los docentes, respecto a la experiencia vivida durante la jornada, al finalizar el taller se aplicó una encuesta con preguntas abiertas, cuyas respuestas mostraron los intereses, las dificultades y el nivel de satisfacción de los participantes. Algunas de las respuestas proporcionadas por los docentes son las siguientes.

En cuanto a las razones para capacitarse mencionaron:

- "por el contexto que viven los alumnos" (docente de Diseño de Vestuario y Textil).

- "para brindar lo mejor a los alumnos" (docente de Pedagogía en Educación General Básica).

- "porque la tecnología es un recurso práctico" (docente de Diseño de Interiores y Exteriores).

- "para sistematizar el intercambio de información" (docente de Ingeniería Comercial).

- "para acompañar los procesos de práctica" (docente de Diseño Gráfico).

- "para mejorar mi labor docente" (docente de Educación Parvularia).

Las razones expresadas respecto a la capacitación dan cuenta que los académicos valoran positivamente el aporte de las TIC en su labor docente. Por otra parte, algunas expresiones utilizadas para justificar la integración de la tecnología a la labor docente son las siguientes:

- "es el mundo en que vivimos" (docente de Diseño de Vestuario y Textil).

- "permite consolidar los conocimientos" (docente de Relaciones Públicas).

- "es útil" (docente de Publicidad).

- "aumenta los recursos para el alumno" (docente de Pedagogía en Educación General).

- "permite actualizar la formación de nuestros alumnos respecto a la sociedad en que vivimos" (docente de Trabajo Social).

- "nos permite innovar y mejorar nuestra labor" (docente de Pedagogía en Educación General). 
Según las opiniones expresadas, los docentes justifican la integración de la tecnología en sus prácticas pedagógicas. En cuanto a las dificultades percibidas por los docentes en la implementación de este proyecto, destacan:

- "No sé cómo sacar provecho útil al aula" (docente de Agronomía).

- "Nunca he usado el computador para mis clases" (docente de Medicina Veterinaria).

- "No sé subir información" (docente de Periodismo).

Las dificultades enunciadas evidencian dos grupos: docentes sin y con competencias tecnológicas que sólo necesitan familiarizarse con la herramienta, información valiosa que optimiza el proceso.

Finalmente, entre las sugerencias planteadas por los docentes destacan:

- "Realizar talleres en vacaciones, para contar con suficiente tiempo para programar y preparar las actividades en el aula virtual" (docente de Trabajo Social).

- "Hacer una capacitación directa a los estudiantes, para que fuera más cercana la adhesión" (docente de Diseño de Vestuario).

- "Sería bueno que después de un tiempo, se hiciera un nuevo taller para aclarar dudas más particulares" (docente de Dirección y Producción de Eventos).

Las sugerencias enunciadas por los académicos fueron un aporte para mejorar la ejecución del programa.

\subsection{Tercera etapa: experimentación}

La práctica individual fue otro de los elementos preponderantes en el proceso; a través de ella se logró la confianza y seguridad en el manejo de la herramienta, condición necesaria para el uso pedagógico. Para evitar la frustración y desmotivación en el momento de interactuar en forma personal con el aula, se diseñó un instructivo, al que podían recurrir en caso de olvidar el procedimiento, y se preparó un archivo de preguntas frecuentes. De este modo, contaban con el recurso para resolver las dificultades. 
Posterior a la capacitación, se realizó el seguimiento de cada participante. Mediante correo electrónico se estableció un contacto personal para ofrecer ayuda y colaboración en el uso de la herramienta. Esta iniciativa ayudó a crear un vínculo que generó un ambiente de acompañamiento, estimulando a continuar practicando y experimentando en el aula de su asignatura.

En los casos de docentes, que no contaban con las competencias tecnológicas básicas, se necesitó mayor atención, realizando sesiones personalizadas que les permitieron vencer el temor.

\subsection{Cuarta etapa: retroalimentación}

Para mantener la motivación y evitar el desencanto se diseñó un plan de apoyo basado en la comunicación constante con el docente. Consistió en monitorear diariamente cada una de las aulas, observando progresos y dificultades. Al mostrar un avance, se enviaba un mensaje de congratulación, resaltando el hecho. En cambio, al advertir un error del docente en el uso de la herramienta se le corregía, adjuntando un video tutorial y un archivo de ayuda.

En los docentes que no presentaban problemas en el uso instrumental se realizaba un apoyo pedagógico, es decir, se enviaban sugerencias de recursos con ejemplos de actividades relacionadas con las unidades de la asignatura, anticipándose a la inquietud "icómo lo hago?"

El soporte se realizaba a través de correo electrónico, atención telefónica y reuniones personalizadas. Para incentivar el desarrollo de competencias y habilidades propias de la educación superior contemporánea, se creó el "Salón Virtual de Académicos", integrado por distintas secciones, entre ellas: "Capacitación y Formación", "Archivos de Ayuda", "Recursos web 2.0", "TIC en el aula". Además incluía un "Foro Consultas" para aclarar dudas, y también un "Foro Sugerencias", donde los docentes compartían recursos, actividades y experiencias con sus pares. 


\section{Algunos cambios observados a partir de la experiencia}

\subsection{La implementación y los docentes}

Algunos cambios se han expresado de manera paulatina a partir de la implementación del proyecto en cuanto a su uso general, instrumental y pedagógico.

Respecto al uso general de la plataforma, a dos años de haber iniciado el programa se observa un aumento progresivo y significativo manifestado en la cantidad de aulas virtuales. En el año 2008 de 22 $(5,2 \%)$ docentes que usaron el aula virtual, la cifra aumentó a 162 en el año 2009 (38,6\%), de un total de 419 profesores que impartían clases en pregrado. En consecuencia, el número de asignaturas con aulas virtuales creció de 30 (3,8\%) en el año 2008 a 318 (34,7\%) en el año 2009, de un total de 865. Por tanto, también aumentó la cobertura de los alumnos. En el año 2008 de un universo de 1.170 estudiantes, 250 (21,3\%) utilizaron aulas virtuales, en cambio, en el año 2009, fueron 450 estudiantes $(33,5 \%)$ de un total de 1.340 .

La evolución del uso instrumental de las aulas virtuales se manifestó a través del aumento en la variedad de recursos. En primera instancia, los docentes subieron archivos a la plataforma, los más comunes fueron el programa de la asignatura, la calendarización y algunos documentos de apoyo, principalmente presentaciones Power Point. A continuación se incorporaron otros, como videos y software. Más tarde instalaron enlaces a sitios web de apoyo.

A fines del año 2009 se comenzó a masificar el foro llamado "Novedades", herramienta que el docente usaba para transmitir información y/o comunicación de sucesos relacionados con la asignatura. Después se registraban fechas de pruebas, trabajos, talleres, visibles en el calendario que posee el aula. Finalmente se instaló el uso de directorios o carpetas para organizar los archivos por temas.

Con relación al uso pedagógico de la herramienta, en el año 2009 se constató que aumentó el número de docentes que incorporaron actividades de aprendizaje, como tareas y foros, situación 
que favoreció el ingreso y la actividad del alumno, porque los obligó a interactuar con la plataforma para cumplir con lo solicitado.

Al principio existían sólo foros generales, donde el profesor exponía un tema y los estudiantes intervenían con un comentario u opinión. Se diseñó un archivo de ayuda pedagógica llamado "Estrategias" para crear un foro didáctico, que se publicó en "Salón de Académicos" y se envió por correo electrónico para optimizar el uso pedagógico de este recurso.

Posteriormente apareció el cuestionario, algunos lo usaban como actividad de aprendizaje y otros como instrumento de evaluación en línea. En seguida surgieron algunos casos (docentes con mayor dominio tecnológico) que incorporaron en sus aulas virtuales presentaciones interactivas, sitios web, videos, software de apoyo, blog y facebook, WebQuest, blog y Wiki.

Finalmente podemos revisar y analizar algunos testimonios de académicos levantados a partir de esta experiencia, y que dan cuenta de distintos aspectos positivos valorados por ellos. Así, por ejemplo, se puede constatar que la plataforma de aulas virtuales es valorada en tanto medio de comunicación entre profesores y alumnos:

"Mi experiencia con el aula virtual es que ésta se ha transformado en un excelente medio de comunicación con mis alumnos, ellos están acostumbrados al uso de la red para obtener información, por lo cual se familiarizan rápidamente con la interfaz, y valoraron el aula como un medio de comunicación ordenado y confiable, estos valores se extrapolan a la práctica docente, ya que los alumnos entienden que es el profesor quien administra la plataforma y la mantiene actualizada" (docente Diseño Gráfico).

"El aula virtual permite la interacción entre profesor y alumno y entre alumnos a través del chat, foros o correo electrónico posibilitando el intercambio de experiencias, y el enriquecimiento y desarrollo de los distintos temas. Asimismo provee del espacio para que los alumnos reciban y/o envíen sus tareas al profesor en los plazos 
establecidos y luego que este último pueda leer, corregir y evaluar por este mismo medio" (docente de Ingeniería Comercial).

También se levantaron testimonios que dan cuenta de la herramienta como facilitadora de la relación pedagógica entre profesores y alumnos:

"En la red, el silencio se siente... se ve y es posible de abordar privadamente...en un tiempo más allá de la sesión de clases. En este sentido, vincularme con mis alumnos en un tiempo en que tengo la disposición mental y el tiempo necesario, ha derivado en una notable mejoría de la relación pedagógica produciendo un impacto, un resultado cuantificable al observar la evaluación que los alumnos han hecho de mi trabajo docente" (docente Trabajo Social).

A su vez, es valorada como estímulo para el desarrollo de nuevos proyectos dentro de la trayectoria académica de los docentes:

"Cuando me enfrenté por primera vez al aula virtual comencé tímidamente a subir mis apuntes, luego a realizar una prueba, y finalmente trabajé durante un año en un proyecto que hace años quería hacer. Crear un material con información de textiles, y ahí es donde surge el ¿cómo hacerlo?, y la respuesta fue inmediata: hacerlo digital para ser utilizado en el aula virtual por todos los alumnos de la carrera. Y ahora me encuentro apasionada diseñando un curso interactivo de enseñanza de teoría textil a través de internet. Uno nunca sabe las vueltas que da la vida......" (docente de Vestuario y Textil).

Asimismo observa que los docentes perciben que el trabajo con esta plataforma les permite adquirir nuevas competencias y por ende mejorar el currículum:

"Hace poco más de dos años atrás, recibimos la noticia que la universidad implementaría el sistema de aulas virtuales. Como casi siempre sucede frente a la innovación y el cambio, muchos se mostraron reticentes. Por mi parte, y muy probablemente debido a 
mi ignorancia ante el asunto, y aquí calza perfecto aquel adagio popular que reza que la ignorancia es atrevida, me interesó de inmediato el tema y quise interiorizarme de él cuanto antes. La herramienta que nominamos "Aulas Virtuales" es absolutamente fabulosa. Las razones: una tecnología para la información y el conocimiento rápida, eficiente, limpia, generadora de hábitos de estudio, proactiva, multidisciplinar, sólidos y democráticos. Los alumnos, "nativos digitales", como se los conoce hoy en día, al igual que los mayores, tampoco son tan proclives al cambio, siempre tienen excusas para dilatar los inicios, pero una vez que conocen y se han familiarizado con el instrumento, agradecen y reconocen sus múltiples beneficios" (Docente de Diseño).

Y finalmente, vale la pena mencionar que algunos docentes perciben la herramienta como un facilitador de su trabajo en general:

"Contar con las TIC me ha facilitado mi trabajo, ya que al integrar novedosas y efectivas técnicas, el estudiante se siente acogido y le permite participar activamente. Hay sentido de pertenencia con sus compañeros, logrando un resultado de un trabajo colaborativo" (docente de Dirección y Producción de Eventos).

"El uso del aula virtual ha sido una herramienta de apoyo bastante útil en mis ramos, como mecanismo de comunicación es una plataforma muy confiable y permite ordenar la información y respaldar los comunicados en forma pública para el grupo curso. Además facilita el apoyo a las clases permitiendo subir información, vincular sitios web, entre otras. La herramienta que más he utilizado es la posibilidad de subir tareas por parte de los alumnos, esto facilita el control de entregas. En general, para mí es un apoyo en comunicación, control de evaluaciones y publicación de material de apoyo. Una muy buena herramienta" (docente de Agronomía).

"Las aulas virtuales no son solo un medio comunicacional sino como parte fundamental facilitadora y vinculante de los aprendizajes, situación que he podido vivenciar con 
claridad en mis cursos de Historia Antigua y actualmente en la asignatura Fundamentos de la Educación e Investigación Historiográfica que estoy realizando este primer semestre de 2010. La Unidad de Tecnología Educativa junto con la biblioteca vendrían a ser pilares, nuestro trabajo disciplinario" (docente de Pedagogía en Historia y Ciencias Sociales).

\subsection{La implementación del proyecto y los estudiantes}

El uso del foro Novedades y el correo electrónico mejoró la comunicación entre los alumnos y entre ellos y los docentes. La publicación de material de apoyo facilitó el acceso a la información y la retroalimentación de las temáticas tratadas en la clase presencial.

A medida que el docente comenzó a agregar actividades, aumentó la participación de los alumnos, asumiendo un rol más protagónico en su aprendizaje. Se percibe que el uso de variados recursos despertó el interés por el ramo, mejorando su compromiso personal y su desempeño en la asignatura.

\section{Consideraciones finales}

El diseño y la implementación de una estrategia institucional hacen posible la inserción de la tecnología y la innovación en las prácticas de los docentes, aunque es un proceso lento.

El uso de la tecnología debe considerar dos etapas: instrumental y pedagógica. La primera requiere de práctica y apoyo constantes para evitar la frustración y la desmotivación. La segunda surge cuando el docente logra seguridad y confianza en la herramienta.

El seguimiento y la colaboración juegan un papel preponderante en el proceso, porque surgen dudas y consultas que requieren de una respuesta inmediata que puede ser aclarada mediante correo electrónico, atención telefónica o entrevista personal, es decir, contar con una mesa de ayuda permanente.

La estimulación constante y el reconocimiento a logros y/o avances mantienen la motivación e incentivan al docente a seguir 
incursionando en el aula. El rol que asume el docente inicialmente es de instructor, pero a medida que va incorporando actividades cambia a facilitador, guía y en algunos casos mediador.

Respecto a los alumnos, a pesar de ser nativos digitales (alumnos que nacieron en la era digital y son usuarios permanentes de Messenger, Facebook, internet, reproductores de audio y video, entre otros) también necesitan un espacio -menor que el requerido por los docentes- para familiarizarse con los recursos de la plataforma.

\section{Referencias bibliográficas}

Marquès, Pere (2001) Algunas notas sobre el impacto de las TIC en la universidad. Revista Educar (28), p. 91.

Salinas, Jesús (2002) Las TIC como medios para una nueva universidad. $2^{\circ}$ Congreso Internacional Docencia Universitaria e Innovación. Tarragona 1-3 julio.

Disponible en http://gte.uib.es/publicacions/CIDUI_salinas.pdf 